\title{
Caracterização, modelagem e simulação de fio atuador de liga de memória de forma
}

\author{
Antônio Júlio Santana Barroso* \\ José Sérgio da Rocha Neto** \\ Eisenhawer de Moura Fernandes ${ }^{* * *}$ \\ * Departamento de Engenharia Elétrica (DEE), \\ (antonio.barroso@ee.ufcg.edu.br). \\ ** Departamento de Engenharia Elétrica (DEE), \\ (zesergio@dee.ufcg.edu.br) \\ *** Departamento de Engenharia Mecânica (DEM), Universidade \\ Federal de Campina Grande, Campina Grande, Paraíba, Brasil \\ (eisenhawer@ee.ufcg.edu.br)
}

\begin{abstract}
The shape memory alloys (SMA) have been extensively studied and used in actuators for several areas in recent years, mainly due to their reliability, good deformation rate by weight with shape recovery, smooth and silent displacements. Despite the many advantages of these actuators, their use for position control faces difficulties in the characterization of parameters due to their variation in time, and consequently in the modeling of the systems. The present work has the objective of characterizing and quantifying the most important parameters of the material, mathematically modeling the plant composed of a cantilever beam, whose deformation is measured at the fixed end, actuated by the shape memory alloy wire, and simulate in software the model obtained for comparison with results of the real plant, aiming in the future the application of a closed-loop control system.

Resumo: As ligas de memória de forma (LMF) têm sido bastante estudadas e utilizadas em atuadores para diversas áreas nos últimos anos, principalmente devido a sua confiabilidade, ótima relação deformação por peso com recuperação de forma, deslocamentos suaves e silenciosos. Apesar das diversas vantagens desses atuadores, o seu uso para controle de posição enfrenta dificuldades na caracterização de parâmetros devido a sua variação no tempo, e consequentemente na modelagem dos sistemas. O presente trabalho tem como objetivo caracterizar e quantificar os parâmetros mais importantes do material, modelar matematicamente a planta composta por uma viga engastada, cuja deformação é medida na extremidade fixa, atuada pelo fio de liga de memória de forma, e simular o modelo obtido em software para comparação com resultados da planta real, visando futuramente a aplicação de um sistema de controle em malha fechada.
\end{abstract}

Keywords: Intelligent Actuators, SMA; Characterization; Modeling; Simulation.

Palavras-chaves: Atuadores Inteligentes; LMF; Caracterização; Modelagem; Simulação.

\section{INTRODUÇÃO}

As Ligas de Memória de Forma (LMF) são utilizadas na instrumentação eletrônica, principalmente no desenvolvimento de atuadores, devido ao seu Efeito de Memória de Forma (EMF). Este efeito consiste na capacidade de retornar à uma forma preestabelecida com o aumento de temperatura até um valor crítico, chamado de temperatura de transformação reversa (J. Jayender and Ostojic, 2008). Essas ligas especiais podem absorver e dissipar energia mecânica através de uma mudança de forma histerética reversível quando submetido à uma carga cíclica mecânica, o que as tornam populares para detecção e atuação, absorção de impacto e aplicações de amortecimento e vibração. Entretanto, o seu uso é restrito à aplicações em baixas frequências $(0,1$ a $20 \mathrm{~Hz})$. Respostas em alta frequência são possíveis para a classe de ligas com memória de forma magnética (Lagoudas, 2008).
Esses materiais apresentam uma ótima relação deformação por peso, deslocamentos suaves e silenciosos, sendo utilizados como sensores e atuadores em diversos setores industriais como aeroespacial, automotivo, biomédico, odontológico e exploração de petróleo (Lagoudas, 2008), fornecendo dispositivos simples, compactos e confiáveis (Yamauchi et al., 2011). Quando utilizadas em atuadores termomecânicos, como neste trabalho, onde o aquecimento é realizado por Efeito Joule através da passagem de corrente, essas ligas tornam-se uma boa alternativa para geração de grandes forças e deformações com baixas frequências (Lima, 2008).

Utilizando informações mais precisas sobre algumas propriedades importantes do material provenientes de ensaios mecânicos, juntamente com outras retiradas da literatura (Inc., 2018), (J. Jayender and Ostojic, 2008), (Velázquez and Pissaloux, 2012), (Abdelaal and Nagib, 2014), apresenta-se aqui uma alternativa de modelagem 
analítica para o atuador de fio de LMF que permita a aplicação futura de um sistema de controle simples em malha fechada, utilizando técnicas de controle clássicas, como controladores PI ou PID, com índices de desempenho superiores à controladores obtidos através de uma modelagem por identificação de sistemas (Campos, 2018).

\section{PROPRIEDADES DAS LMF'S}

As LMF's possuem propriedades ímpares definidas pela sua estrutura cristalina. Elas possuem duas fases com diferentes estruturas e propriedades: austenita em altas temperaturas, e martensita em baixas temperaturas. A transformação entre as fases ocorre pela distorção da estrutura de cisalhamento (transformação martensítica), onde cada cristal martensítico pode ter duas variantes de orientação diferentes: martensita desorientada $\left(M^{d}\right)$, com variantes autoacomodados, e martensita orientada $\left(M^{o}\right)$, com um variante dominante (Lagoudas, 2008). A transformação da austenita para martensita é dita transformação direta, e da martensita para austenita como transformação inversa.

\subsection{Efeito de memória de forma}

O Efeito de Memória de Forma (EMF) é a capacidade de recuperação de forma do material previamente estabelecida através da variação de temperatura, após sofrer deformações plásticas em uma temperatura específica que exceda o limite elástico do material (Elahinia, 2015).

Esse fenômeno pode ocorrer em caminho simples (one way), onde o material deformado mecanicamente recupera a sua forma original quando aquecido, porém quando resfriado não consegue retornar à sua forma deformada original, sendo necessária uma força para deformá-lo novamente, e também em caminho duplo (two-way), onde o material memoriza duas formas diferentes, uma para baixas temperaturas e outra para altas temperaturas.

Uma LMF inicialmente na fase fria (martensita desorientada) ao sofrer uma aplicação de tensão mecânica, tem suas variantes microscópicas reorientadas, transitando para fase de martensita orientada, que é mantida mesmo após retirar a carga aplicada. Aquecendo-se acima da temperatura final da austenita $\left(A_{f}\right)$, ocorre a transformação completa para fase de austenita, onde temos a recuperação de forma e geração de força no fio, que se contrai devido a organização do tipo cúbica de face ou corpo centrados (CFC/CCC) do material. Ao resfriar até uma temperatura menor que $M_{f}$ (temperatura final da martensita), ela retorna à fase inicial de martensita desorientada. Caso o resfriamento ocorra ainda com a carga aplicada, tem-se uma transformação direta da austenita para martensita orientada, e caso aquecido novamente, esse recupera sua forma original de austenita (Lagoudas, 2008).

\section{PLATAFORMA EXPERIMENTAL}

Neste trabalho utilizou-se uma plataforma experimental para testes que possui um fio de LMF (NiTiNOL do tipo two-way) como atuador no controle de posição e deformação da extremidade fixa de uma viga em balanço. Essa plataforma foi inicialmente desenvolvida por Lima (2008) e posteriormente utilizada e aperfeiçoada por Campos (2018).

Sua funcionalidade é controlar a posição da viga entre valores estabelecidos pelo usuário, utilizando a medição da deformação na extremidade fixa da viga através de sensores (extensômetros) e utilizando um fio de LMF como atuador. A viga é deformada pelo seu próprio peso em balanço e por uma carga externa facultativa, consequentemente esticando o fio. Aplicando-se uma potência elétrica no atuador, recupera-se a forma original mediante aquecimento, atuando força suficiente na viga para retorna-la a sua posição desejada.

A estrutura física de projeto da plataforma é apresentada nas Figuras 1 e 2. Essa configuração foi desenvolvida para experimentos de modelagem e controle, porém inspira possíveis aplicações na engenharia, no controle de vigas engastadas.

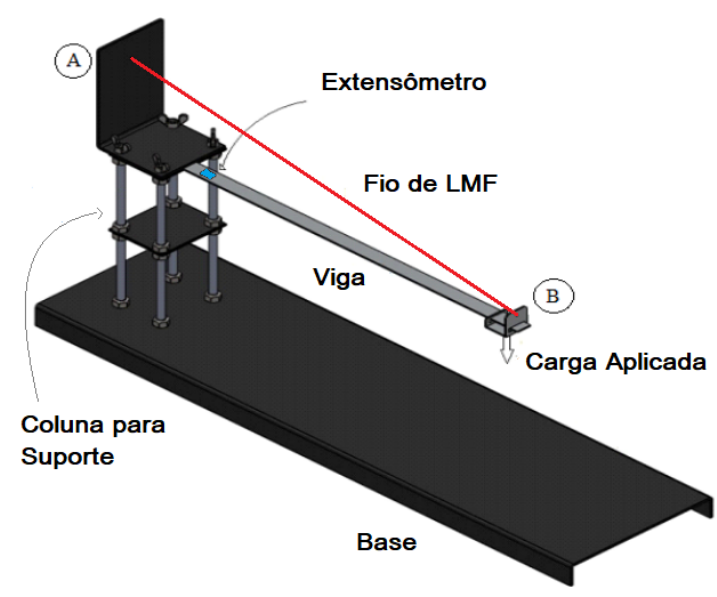

Figura 1. Vista isométrica da plataforma.

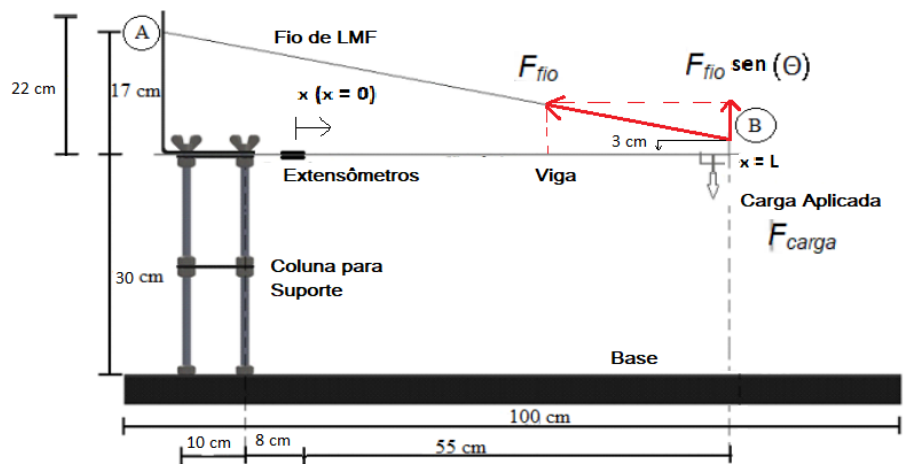

Figura 2. Diagrama de corpo livre do sistema (vista lateral).

\section{CARACTERIZAÇÃO E MODELAGEM}

\subsection{Ensaios do Material}

Com o intuito de esclarecer incertezas nas informações sobre certos parâmetros do material (fio de LMF) apresentados na literatura (J. Jayender and Ostojic, 2008), (Lagoudas, 2008), (Campos, 2018), foi necessário realizar alguns ensaios mecânicos. Os equipamentos foram fornecidos pelo Laboratório de Materiais e Estruturas Ativas (LaMMEA), pertencente a Unidade Acadêmica de Engenharia Mecânica da UFCG. 
Calorimetria Diferencial de Varredura (DSC): ' É utilizada para obtenção das temperaturas de transformação de fase do material, parâmetros fundamentais na modelagem do sistema. As temperaturas podem ser obtidas analisando-se o comportamento de outras propriedades em função da temperatura, como resistência elétrica $R_{L M F} \mathrm{e}$ calor específico $c_{p}$, pois elas variam de forma específica na mudança de fase.

O ensaio consiste em medir o fluxo de calor absorvido ou liberado por uma amostra do fio durante aquecimento e resfriamento à uma taxa constante de variação de temperatura $\left({ }^{\circ} \mathrm{C} / \mathrm{min}\right)$. O resultado é apresentado na Figura 3 .

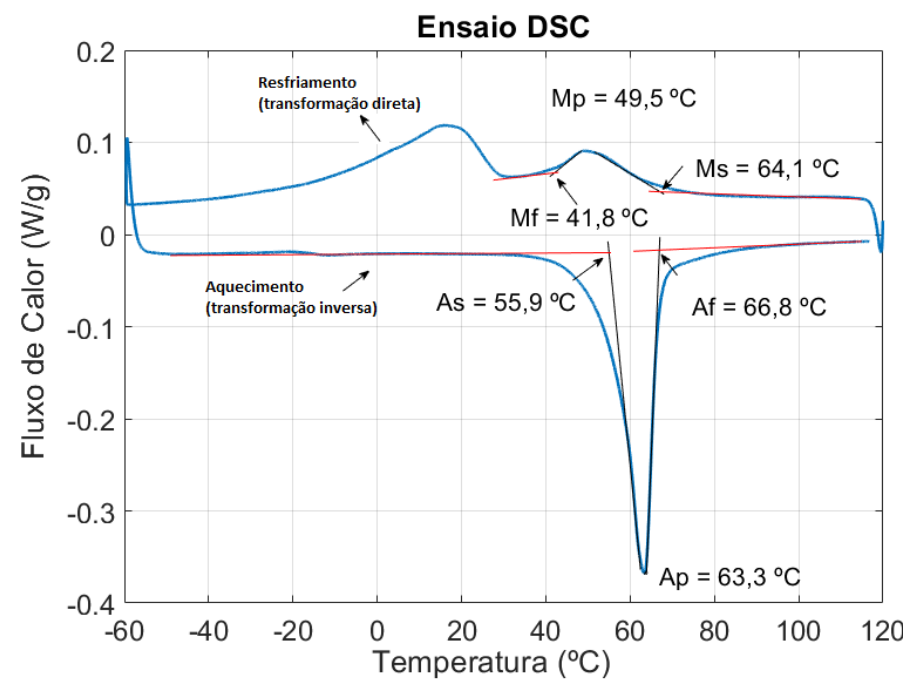

Figura 3. Resultado do ensaio DSC.

As temperaturas de transformação de fase são obtidas pelo método da tangente, utilizando os picos de transformação como referência. São apresentadas também as temperaturas de pico em cada fase $\left(M_{p}\right.$ e $\left.A_{p}\right)$.

Considerando uma temperatura ambiente de $22{ }^{\circ} \mathrm{C}$, observa-se que a LMF utilizada neste trabalho possui temperaturas de transformação de fase acima da temperatura ambiente, indicando que pode-se atingir a contração máxima $(5 \%)$ do fio através da aplicação de potência elétrica. A recuperação de forma é possível através da carga aplicada pela viga engastada e por sua resistência elástica à temperatura ambiente (resfriamento natural).

Análise Mecânica Dinâmica (DMA): Para avaliar os módulos de elasticidade do material em cada fase (Martensita e Austenita) em função da temperatura, foi realizada uma Análise Mecânica Dinâmica (DMA) com equipamento DMA Q800 da fabricante TA Instruments. O ensaio é do tipo tração e consiste na aplicação de uma força oscilatória em uma amostra e o estudo da resposta do material a esta carga aplicada.

Metais comuns apresentam redução do módulo de elasticidade com o aumento de temperatura, porém para as LMFs existe um aumento deste módulo durante a transformação de fase indireta (aquecimento), onde a austenita apresenta uma maior rigidez, conforme apresentado na Figura 4.

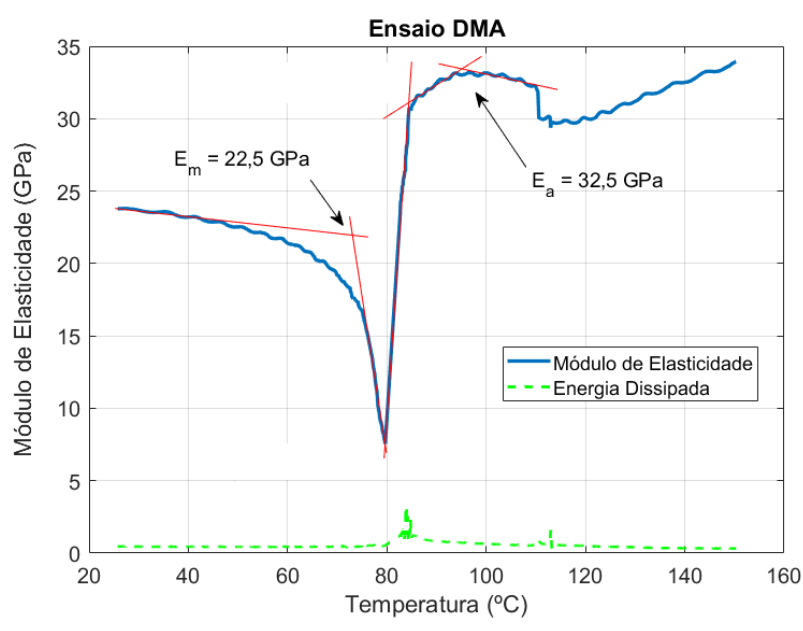

Figura 4. Resultado do ensaio DMA.

Esse ganho de rigidez, consequentemente aumento do módulo de elasticidade e amortecimento, permite aos atuadores de LMF serem utilizados para minimizar vibrações mecânicas em equipamentos industriais. No entanto, é preciso atentar para o aquecimento excessivo do atuador, pois já na região totalmente austenítica (temperaturas acima de $110{ }^{\circ} \mathrm{C}$ ), existe uma redução da capacidade de absorção de energia e, consequentemente, da capacidade de amortecimento da LMF.

Esse ensaio também permite analisar a tensão mecânica aplicada à amostra e os seus valores correspondentes de deformação, em função da temperatura, como também uma relação entre eles, sendo úteis para comparativo e orientação para as simulações computacionais dos modelos e para garantir a utilização do fio dentro do limite de segurança, evitando danos ao material.

\subsection{Modelagem Matemática}

A modelagem do atuador de fio de LMF pode ser dividido em: modelo térmico, modelo da mudança de fase e modelo do sistema mecânico. Nesse trabalho, acrescenta-se ainda um submodelo da Viga engastada que é parte fundamental da plataforma experimental. Isso pode ser visto na Figura 5 .

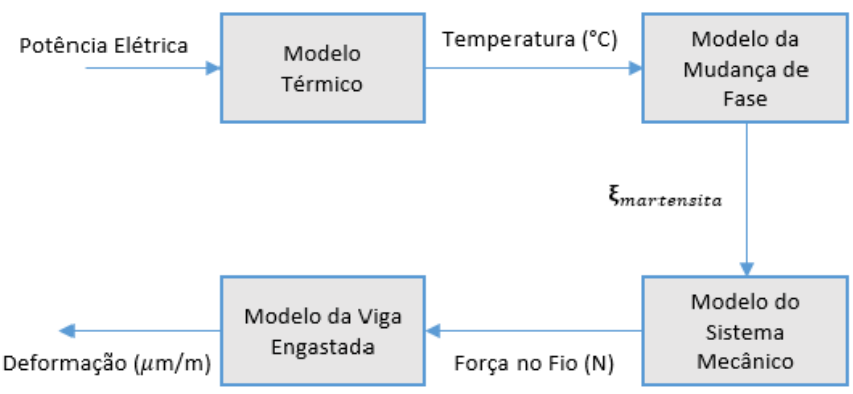

Figura 5. Diagrama de blocos do modelo de atuador LMF.

Uma potência elétrica aplicada fornece uma corrente elétrica ao fio de LMF, aquecendo-o por efeito Joule, elevando sua temperatura $(T)$. Em seguida, o material sofre uma mudança de fase na sua estrutura microscópica, sendo 
caracterizada pela sua porcentagem de martensita $(\xi)$ presente, sendo $\xi=1(100 \%)$ para a sua fase fria (apenas martensita) e $\xi=0(0 \%)$ para a sua fase quente (apenas austenita). Essa fração determina a deformação do fio e força aplicada por ele à viga engastada, onde é medida a deformação $\varepsilon_{v i g a}$.

Modelo Térmico: O atuador de fio com LMF tem seu princípio fundamentado na variação de temperatura para gerar forças e deformações, e que a presente estrutura da plataforma não possui medição de temperatura do fio $T$, é necessário primeiramente definir o seu modelo térmico. $\mathrm{Na}$ literatura é possível encontrar essa modelagem bem definida e explorada, como em Abdelaal and Nagib (2014), D and Dhanalakshmi (2017), Nascimento et al. (2009), Tai and Ahn (2012), Velázquez and Pissaloux (2012) e Xiaoguang et al. (2017).

O modelo aqui utilizado é constitutivo e adaptado da literatura, obtido pelo balanço térmico no fio. O calor no fio $\left(Q_{L M F}\right)$ é proporcional a diferença entre o calor fornecido pela fonte externa $\left(Q_{\text {fonte }}\right)$ e as dissipações por convecção $\left(Q_{\text {convec }}\right)$, condução $\left(Q_{\text {cond }}\right)$ e radiação $\left(Q_{\text {rad }}\right)$. O calor dissipado por radiação pode ser desprezado $\left(Q_{\text {rad }} \approx 0\right) \mathrm{em}$ aplicações na atmosfera, assim como o calor dissipado por condução $\left(Q_{\text {cond }} \approx 0\right)$, ao considerar a temperatura atual constante ao longo todo o fio, como neste trabalho.

A temperatura do fio $T$ é determinada por uma equação diferencial de primeira ordem (2) a partir da Equação (1):

$$
\begin{aligned}
& Q_{L M F}=Q_{\text {fonte }}-Q_{\text {convec }}-Q_{\text {cond }}-Q_{\text {rad }} \\
& Q_{L M F}=Q_{\text {fonte }}-Q_{\text {convec }}
\end{aligned}
$$

$$
m_{L M F} \cdot c_{p} \cdot \frac{d T}{d t}=P_{L M F}-h \cdot S\left(T-T_{a m b}\right)
$$

onde $m_{L M F}$ é a massa do fio, $P_{L M F}$ é a potência elétrica fornecida pela fonte, $h$ é o coeficiente de convecção, $S$ é a área superficial do fio e $T_{a m b}$ é a temperatura ambiente.

Os parâmetros do modelo térmico estão reunidos na Tabela 1 .

\begin{tabular}{lll}
\hline Parâmetro & Valor & Fonte \\
\hline$d$ & $0,2 \mathrm{~mm}$ & Experimental \\
$l$ & $789 \mathrm{~mm}$ & Experimental \\
$S$ & $4,96 \cdot 10^{-4} \mathrm{~m}^{2}$ & Experimental \\
$V$ & $2,48 \cdot 10^{-8} \mathrm{~m}^{3}$ & Experimental \\
$T_{a m b}$ & $22^{\circ} \mathrm{C}$ & Experimental \\
$m_{L M F}$ & $1,58 \cdot 10^{-4} \mathrm{~kg}$ & Experimental \\
$\rho_{L M F}$ & $6.450 \mathrm{~kg} / \mathrm{m}^{3}$ & Fabricante \\
$c_{p}$ & $837 \mathrm{~J} / \mathrm{kg} \cdot \mathrm{K}$ & Fabricante \\
$k$ & $1.800 \mathrm{~W} /(\mathrm{m} \cdot \mathrm{K})$ & Fabricante \\
$R_{L M F}$ & $29 \Omega / \mathrm{m}$ & Fabricante \\
$h$ & $150 \mathrm{~W} / \mathrm{m}^{2} \cdot{ }^{\circ} \mathrm{C}$ & Fabricante \\
\hline
\end{tabular}

Tabela 1. Parâmetros do modelo térmico.

\footnotetext{
Modelo da Mudança de Fase: A LMF possui uma histerese na relação entre a fração martensítica $\xi$ (mudança de fase) e a temperatura $T$. Fisicamente, essa histerese é baseada na dissipação e assimilação do calor latente, devido a mudança de fase, que tende a retardar o aquecimento e o resfriamento.
}

O material possui quatro temperaturas de transição, duas para cada fase (austenita e martensita), e seus valores obtidos através do ensaio DSC são apresentados na Tabela 2 .

O modelo aqui apresentado é baseado em uma relação empírica que utiliza a teoria de estatística Fermi-Dirac, sendo desenvolvida por Liang e Rogers e utilizado em diversos trabalhos, como Abdelaal and Nagib (2014) e Velázquez and Pissaloux (2012), descrevendo a fração martensítica $\xi(T)$ durante o aquecimento (Equação 3) e o resfriamento (Equação 4) do fio em função apenas das temperaturas $M_{s}, M_{f}, A_{s}$ e $A_{f}$, aplicando cargas contantes:

$$
\begin{gathered}
\xi_{a}(T)= \begin{cases}0, & T>A_{f} \\
0,5\left[\cos \left(\pi \frac{T-A_{s}}{A_{f}-A_{s}}\right)+1\right], & A_{s}<T<A_{f} \\
1, & T<A_{s}\end{cases} \\
\xi_{r}(T)= \begin{cases}0, & T>M_{s} \\
0,5\left[\cos \left(\pi \frac{T-M_{f}}{M_{s}-M_{f}}\right)+1\right], & M_{f}<T<M_{s} \\
1, & T<M_{f}\end{cases}
\end{gathered}
$$

onde $\xi_{a}(T)$ e $\xi_{r}(T)$ são as frações martensíticas no aquecimento e resfriamento, respectivamente.

\begin{tabular}{ll}
\hline Temperatura & Valor \\
\hline$M_{f}$ & $41,8^{\circ} \mathrm{C}$ \\
$M_{s}$ & $64,1^{\circ} \mathrm{C}$ \\
$A_{s}$ & $55,9^{\circ} \mathrm{C}$ \\
$A_{f}$ & $66,8^{\circ} \mathrm{C}$ \\
\hline
\end{tabular}

Tabela 2. Temperaturas de transição do fio.

Modelo do Sistema Mecânico: A mudança de fase, expressa pela fração martensítica calculada $\xi$, permite calcular o trabalho mecânico $(W)$ do material e o movimento do fio gerados pela variação de calor (temperatura) (Abdelaal and Nagib, 2014).

A deformação $\varepsilon$ é modelada por uma equação diferencial de primeira ordem bem fundamentada na literatura (Abdelaal and Nagib, 2014):

$$
\varepsilon+\tau_{m e c} \frac{d \varepsilon}{d t}=\varepsilon_{m}[1-\xi(T)]
$$

onde $\varepsilon_{m}$ é a máxima deformação recuperável da LMF, disponível no manual Inc. (2018), e $\tau_{m e c}$ é a constante de tempo mecânica, que depende do diâmetro do fio.

Conforme demonstrado por Xiaoguang et al. (2017), o módulo de elasticidade do fio $E_{L M F}$ é fundamentado em função da fração martensítica $\xi$ e dos módulos de elasticidade das fases de austenita $E_{a}$ e martensita $E_{m}$ :

$$
E_{L M F}=\xi * E_{m}+(1-\xi) \cdot E_{a}
$$

A força no fio $F_{L M F}$ ao se deformar por efeito Joule é dada em função de $E_{L M F}$, da deformação do fio $\varepsilon$ e da área transversal do fio $A_{t}$ calculada:

$$
\begin{aligned}
& \sigma_{L M F}=E_{L M F} \cdot \varepsilon \\
& F_{L M F}=E_{L M F} \cdot \varepsilon \cdot A_{t}
\end{aligned}
$$


As características mecânicas do fio de LMF são apresentadas na Tabela 3.

\begin{tabular}{ll}
\hline Parâmetro & Valor \\
\hline$\tau_{m e c}$ & $1,5 \mathrm{~s}$ \\
$\varepsilon_{m}$ & $5 \%$ \\
$E_{a}$ & $32.5 \mathrm{GPa}$ \\
$E_{m}$ & $22.5 \mathrm{GPa}$ \\
$A_{t}$ & $3,1416 \cdot 10^{-8} \mathrm{~m}^{2}$
\end{tabular}

Tabela 3. Parâmetros mecânicos do material.

Modelo da Viga Engastada: O modelo da viga engastada possui uma relação entre a força resultante $(F)$ na extremidade livre da viga $(x=L)$ à deformação sofrida $\left(\varepsilon_{\text {viga }}\right)$ na extremidade onde foram fixados os extensômetros para medição da deformação $(x=0)$, conforme apresentado na Figura 2.

A viga é feita de aço e possui comprimento útil $L=55 \mathrm{~cm}$, largura $a=26 \mathrm{~mm}$, espessura $b=2 \mathrm{~mm}$ e momento de inércia da seção transversal $I=1,73 \times 10^{-11} \mathrm{~m}^{4}$. A densidade $\rho_{\text {viga }}=7.860 \mathrm{~kg} / \mathrm{m}^{3}$ e módulo de elasticidade $E_{\text {viga }}=20,6 \times 10^{10} \mathrm{~N} / \mathrm{m}^{2}$ são retiradas da literatura para o aço comum (Hibbeler, 2005).

A deformação da viga $\epsilon_{v i g a}$ é dada por:

$$
\begin{aligned}
\varepsilon_{v i g a} & =\frac{6 F L}{a b^{2} E_{v i g a}} \\
\varepsilon_{v i g a} & =\frac{6 L}{a b^{2} E_{v i g a}}\left[F_{L M F} \cdot \operatorname{sen}(\theta)-F_{\text {carga }}\right]
\end{aligned}
$$

onde $\theta$ é o ângulo formado entre o fio e uma linha paralela à viga (conforme Figura 2) e $F_{\text {carga }}$ a carga facultativa aplicada à viga, calculados por:

$$
\begin{gathered}
\theta=t g^{-1}\left(\frac{14 \mathrm{~cm}}{73 \mathrm{~cm}}\right)=11,23^{\circ} \text { ou } 0.1961 \text { radianos } \\
F_{\text {carga }}=\left(m_{\text {viga }}+2 m_{\text {carga }}\right) \cdot g \cdot \frac{L}{2}
\end{gathered}
$$

onde $g$ é a aceleração da gravidade, $m_{\text {carga é a massa da }}$ carga possivelmente aplicada através de pesos de referência disponíveis no laboratório e $m_{v i g a}$ é a massa da viga, calculada por :

$$
m_{v i g a}=\rho_{v i g a} \cdot V_{v i g a}=\rho_{v i g a} \cdot a \cdot b \cdot L
$$

\section{SIMULAÇÃO DO MODELO}

O modelo do sistema físico foi simulado em malha aberta no Simulink, ferramenta do software MATLAB R2018a, conforme mostrado na Figura 6. As entradas são tensão $(V)$ ou corrente $(I)$ elétricas aplicadas, temperatura Ambiente $\left(T_{a m b}\right)$ e carga externa facultativa aplicada na Viga $\left(F_{\text {carga }}\right)$. A saída é a deformação na viga $\varepsilon_{v i g a}$.

Conforme apresentado na Figura 6, o modelo do atuador pode ser dividido em três submodelos:

- Modelo térmico, definido pela equação 2;

- Modelo de mudança de fase, definido pelas equações 3 e 4;

- Modelo do sistema mecânico, definido pela equação 5;

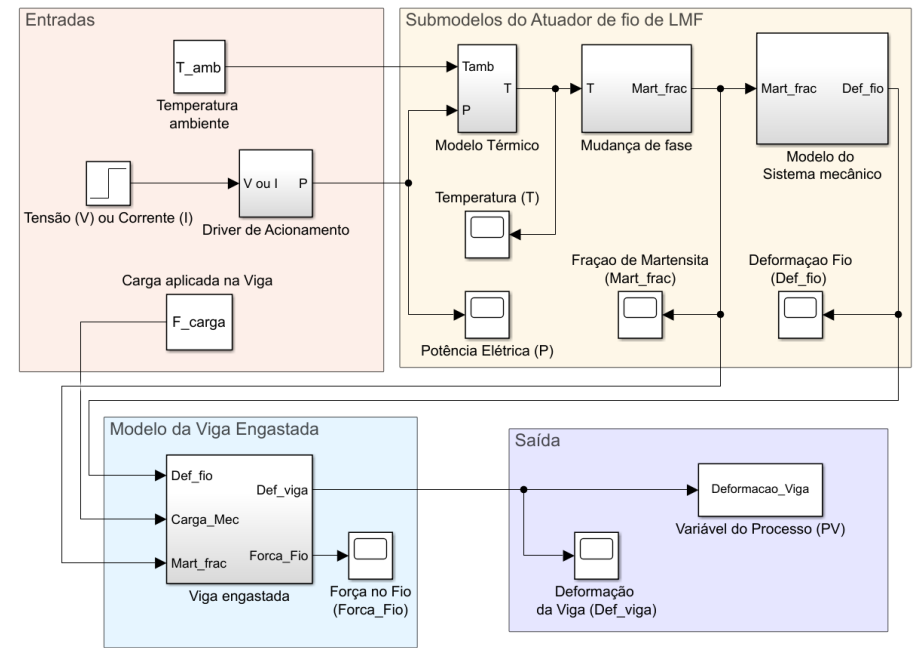

Figura 6. Modelo do sistema em malha aberta.

Além do atuador, modela-se também a viga engastada, fundamental para composição do sistema. Seu modelo é definido pela equação 8 .

\subsection{Validação}

Para avaliar a coerência dos valores de simulação com os valores reais do sistema, é necessário validar os modelos obtidos. Isso foi feito com um comparativo das respostas em malha aberta dos modelos e da planta real através para diversos valores de ciclo (PWM) de tensão aplicada.

O sistema apresenta uma limitação em relação a potência elétrica mínima necessária $(2,4 \mathrm{~W})$, e consequentemente tensão $(7,5 \mathrm{~V})$ e corrente $(320 \mathrm{~mA})$ mínimas, para que a LMF atinja a temperatura $A_{s}$, iniciando a transformação austenítica, e alcance a transformação completa em $A_{f}$, onde ocorre a contração máxima, sendo capaz de produzir força suficiente para deformar a viga conforme desejado, com valores de até $350 \mu \mathrm{m} / \mathrm{m}$.

No driver de tensão, essa tensão mínima equivale a $50 \%$ do ciclo máximo de trabalho do PWM. Foram simulados modelos com valores de entrada entre $60 \%$ e $90 \%$, evitando utilizar o valor máximo para não desgastar o fio, e feito o calculo do erro entre o modelo simulado e a planta real para sinais de entrada iguais. O resultado para a aplicação de 13,5 V (90 \%) é apresentado na Figuras 7 e 8 .

\section{CONCLUSÃO}

O modelo em malha aberta obtido para o sistema é fundamental para futuras implementações de estratégias de controle em malha fechada, a fim de controlar a deformação da extremidade fixa da viga engastada utilizando um atuador de fio com LMF. Ao comparar com resultados experimentais do sistema (planta real), foram obtidos bons índices de erro.

Assim, espera-se que com a aplicação de técnicas de controle clássico (PI/PID) é possível obter controladores com bons índices de desempenho, ou até superiores, em relação aos apresentados em trabalho anterior nesta plataforma (Campos, 2018), onde foi utilizada a identificação de sistemas como estratégia de modelagem (modelo empírico) 

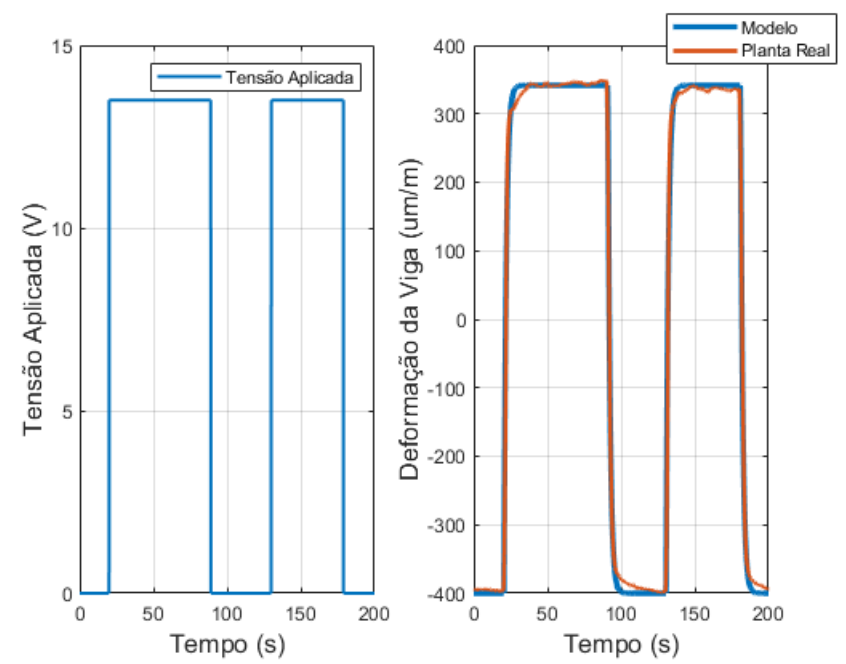

Figura 7. Curvas de entrada e saídas do modelo e planta real, para uma tensão de 13,5 V (90\% do ciclo máximo do PWM).

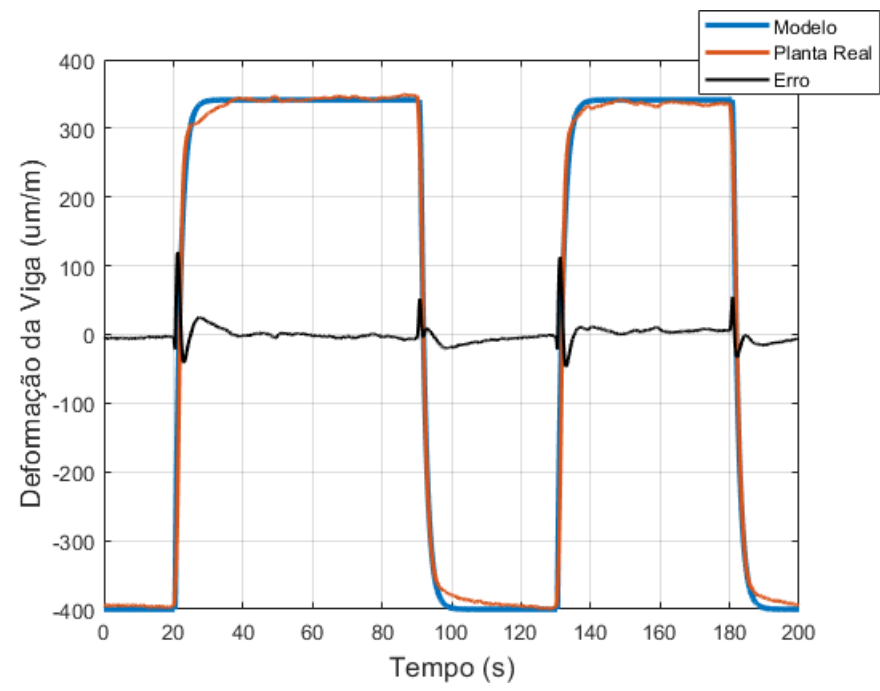

Figura 8. Curvas de saídas e erro entre elas, para uma tensão de 13,5 V (90\% do ciclo máximo do PWM).

e a lógica Fuzzy para ressintonia dos controladores. É esperado que um maior esforço na modelagem permita um menor esforço de controle, o que permite a obtenção de controladores mais simples.

\section{AGRADECIMENTOS}

Os autores agradecem ao Programa de Pós-Graduação em Engenharia Elétrica (PPgEE-COPELE) da UFCG, a Coordenação de Aperfeiçoamento de Pessoal de Nível Superior (CAPES) pelo apoio científico e financeiro, assim como ao Departamento de Engenharia Mecânica da UFCG, especialmente ao pessoal do LaMMEA, pela disponibilidade de equipamentos e materiais para realização deste trabalho.

\section{REFERÊNCIAS}

Abdelaal, W.G.A. and Nagib, G. (2014). Modeling and simulation of sma actuator wire. 9th International Conference on Computer Engineering 85 Systems (ICCES).
Campos, A.H.P. (2018). Estratégias de controle aplicadas a um atuador de liga de memória de forma para o controle de deformação de uma viga engastada. Master's thesis, Universidade Federal de Campina Grande.

D, J.S.R. and Dhanalakshmi, K. (2017). Shape memory alloy wire for force sensing. IEEE Sensors Journal, 17(4), Pag $967-975$.

Elahinia, M.H. (2015). Shape Memory Alloy Actuators, volume 1. John Wiley \& Sons, Ltd, first edition. doi: $10.1002 / 9781118426913$.

Hibbeler, R.C. (2005). Resistência dos Materiais, volume 1. Prentice-Hall, 5th edition.

Inc., D. (2018). Technical characteristics of flexinol actuator wires. URL http://www.dynalloy.com/pdfs/ TCF1140.pdf. Acesso em: 09/10/2018.

J. Jayender, R. V. Patel, S.N. and Ostojic, M. (2008). Modeling and control of shape memory alloy actuators. IEEE TRANSACTIONS ON CONTROL SYSTEMS TECHNOLOGY, 16(2), Pag $279-287$.

Lagoudas, D.C. (2008). Shape Memory Alloys - Modeling and Engineering Applications. Springer, Springer Science+Business Media, LLC, 233 Spring Street, New York, NY 10013, USA, 1st edition.

Lima, W.M. (2008). Plataforma para Análise Comportamental de Atuadores de Ligas com Memória de Forma e para o Controle de Deformação de uma Barra Flexível. Master's thesis, Universidade Federal de Campina Grande.

Nascimento, M., de Araújo, C., de Almeida, L., da Rocha Neto, J., and Lima, A. (2009). A mathematical model for the strain-temperature hysteresis of shape memory alloy actuators. Materials \& Design, 30(3), 551 $-556$.

Tai, N.T. and Ahn, K.K. (2012). Output feedback direct adaptive controller for a sma actuator with a kalman filter. IEEE TRANSACTIONS ON CONTROL SYSTEMS TECHNOLOGY, 20(4), Pag 1081 - 1091.

Velázquez, R. and Pissaloux, E. (2012). Modelling and temperature control of shape memory alloys with fast electrical heating. International Journal of Mechanics and Control, 13(2), Pag 3-10.

Xiaoguang, L., Daohui, Z., Xingang, Z., and Han, J. (2017). Modeling and control of shape memory alloy actuator using feedback linearization. 2017 36th Chinese Control Conference (CCC).

Yamauchi, K., Ohkata, I., Tsuchiya, K., and Miyazaki, S. (2011). Shape memory and superelastic alloys - Technologies and applications. WOODHEAD PUBLISHING, 80 High Street, Sawston, Cambridge CB22 3HJ, UK, 1st edition. 